\title{
A Critical Microscopic Engine in an Optical Tweezers
}

\author{
Falko Schmidt, Alessandro Magazzù \\ Department of Physics, University of Gothenburg, 41296 Gothenburg, Sweden
}

Agnese Callegari

Soft Matter Lab, Department of Physics and UNAM - National Nanotechnology Research Center, Bilkent University, Ankara 06800, Turkey

Luca Biancofiore

Department of Mechanical Engineering, Bilkent University, Ankara 06800, Turkey

Frank Cichos

Peter-Debye-Institute for Soft Matter Physics, Faculty of Physics and Earth Science, University of Leipzig, 04103 Leipzig, Germany

\author{
Giovanni Volpe \\ Department of Physics, University of Gothenburg, 41296 Gothenburg, Sweden \\ giovanni.volpe@physics.gu.se
}

\begin{abstract}
An optically trapped absorbing microsphere in a sub-critical mixture rotates around the optical trap thanks to diffusiophoretic propulsion, which can be controlled by adjusting the optical power, the temperature, and the criticality of the mixture. () 2018 The Author(s)

OCIS codes: (350.4855) Optical tweezers or optical manipulation; (350.4990) Particles
\end{abstract}

The development of microscopic engines capable of converting energy to mechanical work is crucial within the current trend towards nanoscience and nanotechnology [1]. One of the main challenges is that the physics of microscopic engines is fundamentally different from that of macroscopic engines. In particular, microscopic engines have to cope with noise because of the presence of thermal fluctuations. Until now, these fluctuations have been treated mainly as a nuisance with some works studying their statistical properties [2,3] and others tuning their systems in a regime where the consequences of the fluctuations are less relevant [4].

We demonstrate a microscopic engine that is powered by the presence of enhanced fluctuations in the environment [5]. Its working principle is shown in Fig. 1. These enhanced fluctuations emerge because our engine works in a binary mixture (water and lutidine) set close to its critical point where density fluctuations emerge, which can be controlled by the local temperature of the mixture. Using a focused laser beam [6], we optically trap an absorbing microsphere inside this critical binary mixture and we observe that it is set into rotation around the optical axis of the trapping beam due to the emergence of diffusiophoretic propulsion resulting from the presence of enhanced critical fluctuations.
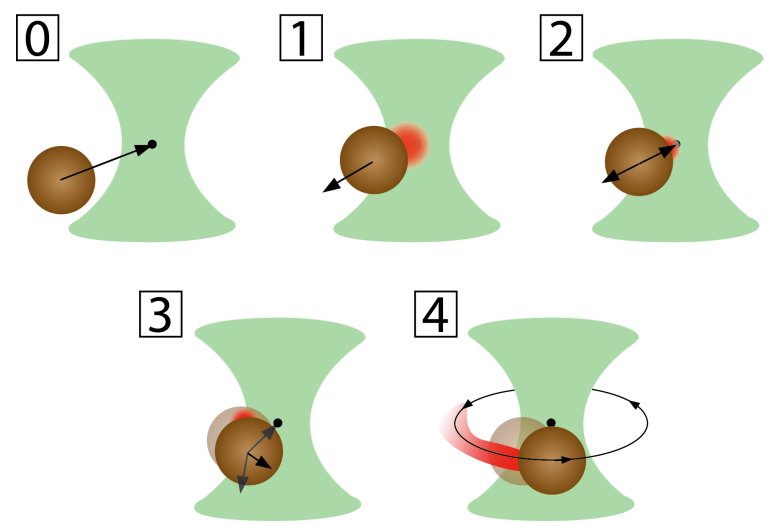

Fig. 2. Working principle of the microscopic critical engine. The critical engine works in several steps: $(0)$ The microscopic particle is attracted towards the center of the optical trap by optical forces (the green shade represents the focused optical beam that creates the optical trap). (1) Some light from the optical trap gets absorbed leading to critical fluctuations (shown in red) that push the particle radially outwards. (2) The particle 
reaches a radial equilibrium where critical forces induced by fluctuations and optical forces cancel out. (3) The presence of inhomogeneities in the particle structure cause local phase separations that lead to a circular motion around the trap center as shown in in (4).

This behavior can be tuned by adjusting the optical power, the temperature and the criticality of the mixture. Increasing the ambient temperature of the system leads first to the rotation of the particle and then to a break-down of the critical engine, as shown in Fig. 2. A similar trend can also be observed when increasing the laser power, so that the optical forces acting on the particle as well as its absorption increase. Using a non-critical mixture (e.g., water) or an off-critical mixture (e.g., a water and 2,6-lutidine mixture with an off-critical composition), we do not observe the emergence of the rotations characteristic of the critical engine.
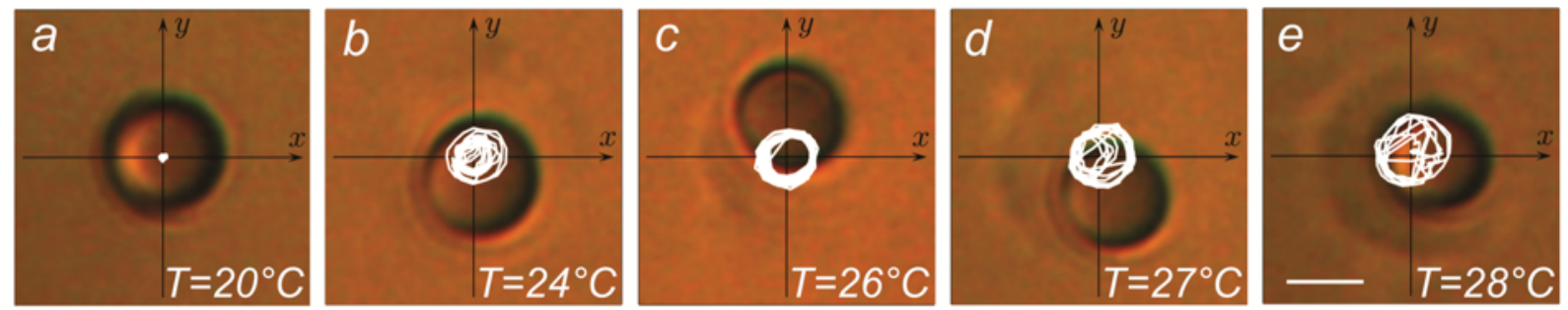

Fig. 2. Realization of a critical microscopic engine using an optically trapped colloidal particle, with radius $R=1,24 \mu \mathrm{m}$, immersed in a water2,6-lutidine critical solution. It is possible to tune the performance of the critical engine by adjusting the ambient temperature. At low temperature

(a) the particle is stably trapped by the optical tweezers. When we start to increase the temperature (b), we can notice that the particle starts to rotate around the optical beam (white solid lines trajectories) reaching its maximum value for a temperature of $26^{\circ} \mathrm{C}$ (c) with a very reproducible trajectory. A further increasing of the temperature results in a decreasing of the engine rotations and performance (d,e).

We have therefore demonstrated a novel kind of microscopic engine that can take advantage of fluctuations to generate rotation and work. Importantly, the fact that this microscopic engine works in a critical mixture does not significantly limit its potential applications. In fact, critical mixtures can be naturally found in many natural systems (e.g., lipid monolayers in cell membranes, the organization of proteins, self-organized criticality in many biological systems) taking advantage of various different order parameters (e.g., density, particle concentration, solution $\mathrm{pH}$ ). Therefore, the microscopic engine we have proposed can find important applications in, e.g., nanotechnology and nanobiomedicine.

[1] O. M. Maragò, P. H. Jones, P. G. Gucciardi, G. Volpe and A. Ferrari, “Optical trapping and manipulation of nanostructures,” Nature Nanotech. 8, 807-819 (2013).

[2] V. Blickle and C. Bechinger. "Realization of a micrometre-sized stochastic heat engine." Nature Phys. 8, 143-146 (2012).

[3] I. A. Martínez, E. Roldán, L. Dinis, D. Petrov, J. M. Parrondo and R. A. Rica, “Brownian carnot engine.” Nature Phys. 12, 67-70 (2016).

[4] P. A. Quinto-Su, “A microscopic steam engine implemented in an optical tweezer.” Nature Commun. 5, 5889 (2014).

[5] F. Schmidt, A. Magazzù, A. Callegari, L. Biancofiore, F. Cichos and G. Volpe, "Microscopic engine powered by critical demixing." Phys. Rev. Lett. 120, 068004 (2018).

[6] P. H. Jones, O. M. Maragò and G. Volpe, Optical Tweezers: Principles and Applications, Cambridge University Press, Cambridge, UK (2015). 